\title{
UVEAL MELANOMA: TUMOUR PHENOTYPE AND METASTATIC POTENTIAL
}

\author{
IAN G. RENNIE \\ Sheffield
}

Uveal melanomas are the commonest intraocular neoplasm in adults. They pose a serious threat to life with up to $50 \%$ of patients ultimately dying as a result of tumour-related disease. ${ }^{1}$ Those patients who succumb to this disease do so not because of the local effects of the primary tumour, but rather because they develop distant metastases that ultimately critically disrupt the function of the affected organs. It is well recognised that the metastatic potential of uveal melanomas is not uniform: some tumours probably have little or no potential to spread, whereas others readily disseminate. These differences in the biological behaviour of these tumours must, at least in part, reflect differences in the phenotypic properties of the tumour cells. At present, our understanding as to what physiological attributes confer a malignant phenotype on these tumours is ill understood.

Metastases develop as a result of a complex multistep process. Tumour cells must first become motile, invade the local extracellular matrix (ECM), and traverse the blood vessel basement membrane and endothelium. Once in the circulation they must evade destruction by the host's immune system. On reaching a distant site, the tumour cells must cross a blood vessel endothelium and basement membrane before, again, invading the local ECM. Finally, the tumour cells must then be capable of unrestrained replication before tangible deposits develop. This process, which is probably highly inefficient, with only a minute fraction of the liberated cells capable of ultimately producing a viable secondary deposit, has been called the metastatic cascade. ${ }^{2}$ The relationship between the tumour cell and the ECM is vital to the metastatic process. Metastatically competent tumour cells must be capable of forming attachments with elements of the ECM, degrading or digesting it and, perhaps, even secreting new matrix components.

Correspondence to: Professor I. G. Rennie, Department of Ophthalmology \& Orthoptics, Royal Hallamshire Hospital, Glossop Road, Sheffield S10 2JF, UK.

\section{ROLE OF ADHESION MOLECULES IN METASTATIC DISEASE}

The determination of the malignant phenotype is heavily dependent upon the cell surface molecules that mediate cell-matrix (and cell-cell) interactions. Adhesion molecules are a heterogeneous group that mediate contacts between the cell and the ECM and can be divided into at least four main groups based on their molecular structure. ${ }^{3}$ These groups are: the integrins, the cell surface proteoglycans, molecules of the immunoglobulin supergene family and lectin-like proteins.

Integrins are the principal receptors on animal cells for binding to most ECM proteins including collagen, fibronectin and laminin. Integrins are transmembrane glycoproteins composed of two chains, alpha and beta, non-covalently associated to form a heterodimeric complex. Approximately 20 integrin heterodimers, made from 14 types of beta subunit and 9 types of alpha subunit, have been defined, and new forms are still being discovered. ${ }^{4}$ This diversity is increased further by the alternate splicing of some integrin RNAs. The normal cell expresses a variety of integrins, which will vary depending on the specific function of the cell. Changes in the expression of cell surface molecules including integrins are probably vital to the acquisition of the metastatic phenotype.

Integrin expression has been studied extensively in cutaneous melanomas. The results, to date, indicate that normal human cutaneous melanocytes express a limited number of b1 and b3 integrins. In contrast, melanoma cells, in vitro and in vivo, can acquire the expression of integrin subunits such as $\alpha 4, \alpha 5$ and $\alpha 7$ which are not normally found on melanocytes. Increased expression of other subunits found on normal melanocytes such as $\alpha 1, \alpha 2$, and $\alpha \mathrm{v}$ may also occur. ${ }^{5-9}$ Moreover, changes in integrin expression in cutaneous melanomas have been observed in the transition from radial to vertical growth phase, with increasing expression of $\alpha 4$ and $\beta 3$ subunits. ${ }^{3,8,10}$ 
Alterations in integrin expression are noted in metastatic lesions. Indeed Moretti et al. ${ }^{3}$ found that the $\alpha 4$ subunit was expressed exclusively in such lesions, suggesting a possible role in mediating the interaction between metastasising cells and vascular endothelium.

In contrast, integrin expression in uveal melanomas has not been studied extensively. ten Berge et al. ${ }^{11}$ examined integrin expression in 32 primary and 4 metastatic uveal melanomas and failed to find any correlation with cell type or invasiveness. Furthermore, they found that the $\alpha \mathrm{v} \beta 3$ vitronectin receptor, which is associated with cutaneous melanoma progression, was not expressed in uveal melanoma. Interestingly, they did find low levels of $\alpha 4$ expression in 3 of the 4 metastatic lesions studied; a finding previously noted in cutaneous metastatic melanomas. ${ }^{3}$ Creyghton et al. $^{12}$ in a study of three cell lines established from uveal melanomas found $\alpha \mathrm{V} \beta 3$ expression in one of them, in contrast to the previous study. Rohrbach et $a l^{13}$ failed to find significant expression of the laminin receptor $(\alpha 6 \beta 1)$, an integrin implicated as an index of invasive potential in other carcinomas, in a study of 12 uveal melanomas. It is clear from these preliminary studies that the expression of integrin molecules in uveal melanomas is variable, and a pattern, if it exists, has yet to be established.

\section{UVEAL MELANOMA CELL ADHESION AND ITS INHIBITION IN VITRO}

Whilst much work has focused on the role of specific adhesion molecules and their possible role in malignant disease, my own group has investigated ways of inhibiting tumour cell adhesion to the ECM by modulating intracellular signal transduction. In particular, we have investigated the possible role of calmodulin antagonists in inhibiting the attachment of uveal melanoma cells to ECM constituents in vitro. ${ }^{14-16}$

Communication between cells is a basic prerequisite of all multicellular organisms. Cell signalling requires both extracellular signalling molecules and a complementary set of receptor proteins on each cell that enable them to bind and respond to each other in a characteristic way. G-protein-linked receptors are the largest family of cell-surface receptors and, in the main, these receptors alter the concentration of one or more intracellular signalling molecules. These molecules are often referred to as intracellular mediators or second messengers. In turn, these intracellular molecules pass on their signal by altering the behaviour of specific cellular proteins.

The two most abundant intracellular mediators are cyclic AMP and calcium ions. In the normal cell the resting concentration of free calcium is only between $10^{-7}$ and $10^{-8} \mathrm{~mol} / \mathrm{l}$, and a 10 - to 100 -fold increase in this concentration initiates the appropriate response. The effect of this increased level of free calcium is propagated further by several specific calcium binding proteins, the most important of which is calmodulin. Calmodulin is a ubiquitous intracellular protein, which comprises a single polypeptide chain of about 150 amino acids with four high-affinity calcium binding sites. It undergoes a conformational change when it binds to calcium. This allosteric change activates the calmodulin which, in turn, is then able to activate a wide variety of enzymes, including those regulating nucleotide metabolism. ${ }^{17}$ In a study of the possible role of signal transduction mechanisms in the acute attachment of murine B16 melanoma cells to ECM proteins, MacNeil et al. ${ }^{25}$ found evidence to suggest that intracellular calcium and calmodulin have significant roles in tumour cell attachment. In contrast, manipulation of cyclic AMP did not appear to affect tumour cell attachment. In the light of these findings, we elected to investigate the effect of calmodulin antagonists, in particular tamoxifen, on human uveal melanoma cells in vitro.

Tamoxifen has been used extensively in the treatment of breast cancer and significantly prolongs the disease-free survival and decreases mortality. ${ }^{19}$ Whilst many of its pharmacological properties are attributable to its anti-oestrogen activity, it also has a significant, albeit lesser effect on patients whose tumours are oestrogen-receptor-negative. Tamoxifen has been used in the treatment of other malignancies, including metastatic cutaneous malignant melanoma, where its effect may not be due to its anti-oestrogen activity. ${ }^{18,20}$ Besides its anti-oestrogen activity, tamoxifen is known to inhibit both calmodulin and protein kinase $\mathrm{C}^{21-23}$ and it is postulated that part of its anti-tumour activity may be mediated via its effect on calmodulin. ${ }^{14}$

To examine the effects of tamoxifen and another calmodulin antagonist J8 ( $\mathrm{N}$-(6-amino-octyl-5-iodo1-naphthalene) on uveal melanoma cell attachment to matrix protein, we established short-term cultures (before passage 6) using a previously described technique. ${ }^{24}$ Cell attachment was measured in 96well, flat-bottomed microtitre dishes that were coated with a specific substrate as described by MacNeil et $_{\text {al. }}{ }^{25}$ Six different cell lines were used and their affinity to attach with a variety of substrates including plastic, type I collagen, type III collagen, type IV collagen, fibronectin, gelatin, RGD, vitronectin and poly-L-lysine was examined to establish a preferred substrate. The cells demonstrated a significant preference for collagens type I, III and IV and fibronectin. ${ }^{15,16}$ The attachment to the substrates was rapid, with cells achieving $90 \%$ attachment on fibronectin (the best substrate) and $60 \%$ attachment on gelatin (the worst substrate) in 10 minutes. The effect of tamoxifen on tumour cell attachment was 
then examined using fibronectin as a substrate. A significant reduction in tumour cell attachment was observed. Three of the uveal melanoma cell lines were then used to test the effect of tamoxifen on attachment to the other previously tested substrates. Again, tamoxifen produced a significant reduction in cellular attachment on all the substrates tested.

Manipulation of the cyclic AMP pathway using forskolin to stimulate adenylate cyclase and dideoxyadenosine (DDA) to inhibit it, failed to inhibit tumour cell attachment significantly. Similarly, attempts to modulate protein kinase $\mathrm{C}$ activity using phorbol ester PMA (stimulatory) and staurosporine (inhibitory) did not significantly affect tumour cell adhesion. Alteration of the intracellular calcium levels using either ionomycin, which increases calcium levels, or TMB8 (3,4,5-trimethoxybenzoic acid 8-diethylamino octyl ester), which reduces intracellular calcium, produced a reduction in cell attachment. J8, a known calmodulin antagonist, also produced a reduction in tumour cell attachment. From these results it may be seen that tamoxifen is capable of reducing tumour cell attachment in vitro and that this effect may be produced by modulation of the calcium/calmodulin pathway.

\section{TUMOUR CELL DEGRADATION OF THE ECM: THE ROLES OF PROTEASES}

Besides adhering to the ECM, tumour cells must be capable of degrading elements of the ECM, including basement membrane, to gain access to the circulatory system. To effect this process, tumour cells are capable of producing a wide range of proteolytic enzymes, including plasminogen activators, metalloproteinases and cathepsins. ${ }^{26,27}$ Two types of plasminogen activator are known to exist: tissue-type plasminogen activator (tPA) and urokinase-type plasminogen activator (uPA). Both forms are capable of converting plasminogen to the active serine protease - plasmin; and both these enzymes may have a significant role in the metastatic process by promoting the degradation of ECM components. ${ }^{27}$ Studies using tumour animal models have shown that the use of antibodies against uPA can either inhibit or reduce the incidence of metastase ${ }^{28,29}$ and an in vitro study using the MeWo human melanoma cell line, which only produces tPA in culture, found that invasion through a variety of matrices was reduced in the presence of an anti-tPA antibody. ${ }^{30}$

Investigations into the possible role of plasminogen activators in ocular melanomas are limited. Cottam et al. ${ }^{31}$ examined human uveal melanoma cell lines for fibrinolytic activity and found it to be present in 9 of the 10 lines studied. Moreover, they found that the highest level of fibrinolysis was present in the cell lines derived from patients with tumours where significant scleral invasion was observed. Recently, Alizadeh et al. ${ }^{27}$ have investigated the role of tissue-type plasminogen activator in the metastasis of murine intraocular melanomas. They used two B16 murine melanoma cell lines: the D5.1G4 line which is known to produce low levels of tPA and has a low level of metastatic activity, and the Queen's line which, in contrast, produces a high level of tPA activity and is known to be highly metastatic. The D5.1G4 line was transfected with a full length of cDNA encoding for human tPA. The Queen's line was transfected with a cDNA which codes for human plasminogen activator inhibitor type 1 (PAI-1). Both transfected and non-transfected cell lines were then inoculated either intravenously or intracamerally into mice which were subsequently killed. The number of pulmonary metastases in each group was determined and it was found that D5.1G4-injected transfected cells produced extensive pulmonary metastases, whilst inoculation with the non-transfected controls did not. In contrast, the transfected Queen's cell line, when injected intravenously, had a significantly reduced metastatic potential compared with the non-transfected controls. Furthermore, the transfected Queen's melanoma cells failed to produce metastases when inoculated intracamerally, whilst the non-transfected cells produced extensive pulmonary deposits. However, intracameral inoculation of the tPA-transfected D5.1G4 cell line did not result in the production of pulmonary metastases. Whilst the results of these experiments are slightly conflicting they do suggest that TPA is involved in the metastasis of murine intraocular melanomas.

Matrix metalloproteinases (MMPs) are a group of enzymes that are involved in normal remodelling processes including embryonic development, postpartum involution and wound healing. They are implicated in a number of pathological processes including rheumatoid arthritis, peridontitis and tumour cell invasion. Enzymes within this group can be distinguished by a number of properties, including a putative zinc binding site, secretion in a zymogen form, cleavage of the enzyme during activation resulting in the loss of approximately $10 \mathrm{kDa}$, cleavage of $\mathrm{ECM}$ components by the enzyme and inhibition by tissue inhibitors of metalloproteinases (TIMPs). ${ }^{26}$ Five subgroups have been designated, and of these the type IV metalloproteinases (gelatinases) have been widely implicated in tumour cell invasion and metastases. ${ }^{26}$ In particular, it has been proposed that secretion of a $92 \mathrm{kDa}$ species of gelatinase (MMP-9) by carcinoma or oncogene-transfected cells correlates with an increased metastatic potential. ${ }^{26}$ Cottam et al. ${ }^{31}$ have investigated the secretion of matrix metalloproteinases in 15 human uveal melanoma lines. They found that all the cell lines secreted a $72 \mathrm{kDa}$ gelatinase (MMP-2) and, in addition, nine of the 
lines secreted a $92 \mathrm{kDa}$ gelatinase (MMP-9). The clinical significance of these results has yet to be elucidated.

\section{CONCLUSIONS}

The dissemination of tumour cells from the eye of a patient with a uveal melanoma to form metastatic deposits in a distant organ is a complex and, probably, highly inefficient multi-step process. To make this journey, the successful tumour cells must be capable of interacting with the ECM of the host. Our understanding of this relationship is, at present, incomplete. The acquisition of this knowledge may allow us to develop new strategies in the fight against this uncommon, but potentially devastating disease.

Key words: Uveal melanoma, Metastases, Cell adhesion, Invasion.

\section{REFERENCES}

1. Egan KM, Seddon JM, Glynn RJ, Gragoudas ES, Albert DM. Epidemiologic aspects of uveal melanoma [review]. Surv Ophthalmol 1988;32:239-51.

2. Fidler IF. Critical factors in the biology of human cancer metastasis: Twenty-eighth G.H.A. Clowes memorial award lecture. Cancer Res 1990;50:6130-8.

3. Moretti S, Martini E, Berti E, Pinzi C, Gianotti B. Adhesion molecule profile and malignancy of melanocytic lesions. Melanoma Res 1993;3:235-9.

4. Alberts B, Bray D, Lewis J, Raff M, Roberts K, Watson JD, editors. Molecular biology of the cell. 3rd ed. New York: Garland Publishing, 1994:995-9.

5. Kramer RH, Vu M, Cheng YF, et al. Integrin expression in malignant melanoma. Cancer Metastasis Rev 1991;10:49-59.

6. Mortarini R, Anchini A. From adhesion to signalling: roles of integrins in the biology of human melanoma. Melanoma Res 1993;3:87-9.

7. Hart IA, Birch M, Marshall JF. Cell adhesion receptor expression during melanoma progression and metastasis. Cancer Metastasis Rev 1991;10:115-28.

8. Albelda SM, Mette SA, Elder DE, et al. Integrin distribution in malignant melanoma: association of the $\beta 3$ subunit with tumour progression. Cancer Res 1990;50:6757-64.

9. Kramer RH, McDonald KA, Vu MP. Human melanoma cells express a novel integrin receptor for laminin. J Biol Chem 1989;264:15642-9.

10. Cheresh DA. Structure, function, and biological properties of integrin $\alpha \mathrm{v} \beta 3$ on human melanoma cells. Cancer Metastasis Rev 1991;10:3-10.

11. ten Berge PJ, Danen EH, van Muijen GN, Jager MJ, Ruiter DJ. Integrin expression in uveal melanoma differs from cutaneous melanoma. Invest Ophthalmol Vis Sci 1993:34:3635-40.

12. Creyghton WM, de Waard-Sledinga I, Danen EHJ, Luyten GPM, van Muijen GNP, Jager MJ. Cytokinemediated modulation of integrin, ICAM-1 and CD44 expression on human uveal melanoma cells in vitro. Melanoma Res 1995;5:235-42.

13. Rohrbach JM, Wild M, Riedinger C, Kreissig I, Thiel HJ. Premetastatic uveal melanoma cells do not express laminin receptors. German J Ophthalmol 1994;3:144-7.

14. MacNeil S, Wagner M, Kirkham EA, Blankson MS,
Lennard MS, Goodall T, et al. Inhibition of melanoma cell/matrix interaction by tamoxifen. Melanoma Res 1993;3:67-74.

15. MacNeil S, Wagner M, Rennie IG. Tamoxifen inhibition of ocular melanoma cell attachment to matrix proteins. Pigment Cell Res 1994;7:222-6.

16. MacNeil S, Wagner M, Rennie IG. Investigation of the role of signal transduction in attachment of ocular melanoma cells to matrix proteins: inhibition of attachment by calmodulin antagonists including tamoxifen. Clin Exp Metastasis 1994;12:375-84.

17. Tomlinson S, MacNeil S, Walker SW, Ollis CA, Merritt JE, Brown BL. Calmodulin and cell function. Clin Sci 1984;66:497-508.

18. Fierro MT, Bertero M, Novelli M, Appino A, Doveil GC, Colonna S, et al. Therapy for metastatic melanoma: effective combination of dacarbazine, carmustine, cisplatin and tamoxifen. Melanoma Res 1993; 3:127-31.

19. Buckley MT, Goa KL. Tamoxifen: a reappraisal of its pharmacodynamic and pharmacokinetic properties and therapeutic use. Drugs 1989;37:451-90.

20. Cocconi G, Bella M, Calabresi F, et al. Treatment of metastatic melanoma with dacarbazine plus tamoxifen. N Engl J Med 1992;327:516-23.

21. Lam H-YP. Tamoxifen is a calmodulin antagonist in the action of cAMP phosphodiesterase. Biochem Biophys Res Commun 1984;118:27-32.

22. Lopes MCF, Value MGP, Carvalho AP. $\mathrm{Ca}^{2+}$-dependent binding of tamoxifen to calmodulin isolated from bovine brain. Cancer Res 1990;50:2753-8.

23. Brian CAO, Liskamp RM, Soloman DH, Weinstein IB. Inhibition of protein kinase $\mathrm{C}$ by tamoxifen. Cancer Res 1985;45:2462-5.

24. Goodall T, Buffey JA, Rennie IG, Benson M, Parsons MA, Faulkner MK, et al. Effect of melanocyte stimulating hormone on human cultured choroidal melanocytes. uveal melanoma cells, and retinal epithelial cells. Invest Ophthalmol Vis Sci 1994;35:826-37.

25. MacNeil S, Wagner M, Wowk I, et al. Intracellular regulation of cell adhesion to extracellular matrix components in murine B16 melanoma cells of differing metastatic potential. Melanoma Res 1992;2:345-54.

26. Cottam DW, Rees RC. Regulation of matrix metalloproteinases: their role in tumor invasion and metastasis. Int J Oncol 1993;2:861-72.

27. Alizadeh $\mathrm{H}$, Ding $M$, Berman M, Bellinham D, Comerford SA, Gething $\mathrm{MH}$, et al. Tissue-type plasminogen activator-induced invasion and metastasis of murine melanomas. Curr Eye Res 1995;13:449-58.

28. Ossowski L, Russo-Payne H, Wilson EL. Inhibition of urokinase-type plasminogen activator by antibody: the effect on dissemination of a human tumour in the nude mouse. Cancer Res 1991;51:274-81.

29. Hearing VJ, Law LW, Carti A, Appella E, Blasi F. Modulation of metastatic potential by cell surface urokinase of murine melanoma cells. Cancer Res 1988;48:1270-8.

30. Meissauer A, Kramer MD, Hofman M, Erkell LJ, Jacob E, Schirrmacher V, et al. Urokinase-type and tissue-type plasminogen activators are essential for in vitro invasion of human melanoma cells. Exp Cell Res 1991;192:453-9.

31. Cottam DW, Rennie IG, Woods K, Parsons MA, Bunning RA, Rees RC. Gelatinolytic metalloproteinase secretion patterns in ocular melanoma. Invest Ophthalmol Vis Sci 1992;33:1923-7. 